\title{
Energy Definition and Dark Energy: A Thermodynamic Analysis
}

\author{
H. Moradpour $\mathbb{D}^{1},{ }^{1}$ J. P. Morais Graça, ${ }^{2}$ I. P. Lobo $\mathbb{D}^{2},{ }^{2}$ and I. G. Salako ${ }^{3}$ \\ ${ }^{1}$ Research Institute for Astronomy and Astrophysics of Maragha (RIAAM), P.O. Box 55134-441, Maragha, Iran \\ ${ }^{2}$ Departamento de Física, Universidade Federal da Paraíba, Caixa Postal 5008, 58051-970 João Pessoa, PB, Brazil \\ ${ }^{3}$ Institut de Mathématiques et de Sciences Physiques (IMSP), 01 BP 613 Porto-Novo, Benin \\ Correspondence should be addressed to H. Moradpour; hn.moradpour@gmail.com
}

Received 19 May 2018; Revised 16 July 2018; Accepted 30 July 2018; Published 9 August 2018

Academic Editor: Elias C. Vagenas

Copyright (C) 2018 H. Moradpour et al. This is an open access article distributed under the Creative Commons Attribution License, which permits unrestricted use, distribution, and reproduction in any medium, provided the original work is properly cited. The publication of this article was funded by $\mathrm{SCOAP}^{3}$.

\begin{abstract}
Accepting the Komar mass definition of a source with energy-momentum tensor $T_{\mu \nu}$ and using the thermodynamic pressure definition, we find a relaxed energy-momentum conservation law. Thereinafter, we study some cosmological consequences of the obtained energy-momentum conservation law. It has been found out that the dark sectors of cosmos are unifiable into one cosmic fluid in our setup. While this cosmic fluid impels the universe to enter an accelerated expansion phase, it may even show a baryonic behavior by itself during the cosmos evolution. Indeed, in this manner, while $T_{\mu \nu}$ behaves baryonically, a part of it, namely, $T_{\mu \nu}(e)$ which is satisfying the ordinary energy-momentum conservation law, is responsible for the current accelerated expansion.
\end{abstract}

\section{Introduction}

Friedmann equations, the ordinary energy-momentum conservation law (OCL) (or the continuity equation), and its compatibility with the Bianchi identity (BI) are the backbone of the standard cosmology which forms the foundation of our understanding of cosmos [1]. Since on scales larger than about 100 Megaparsecs, cosmos is homogeneous and isotropic [1], the FRW metric is a suitable metric to study the cosmic evolution [1]. Relations between thermodynamics and Friedmann equations have been studied in various setups which help us in getting more close to the thermodynamic origin of space-time, gravity, and related topics [2-20].

A thermodynamic analysis can also lead to a better understanding of the origin of dark energy, responsible for the current accelerated universe [15-33]. In fact, there are thermodynamic and holographic approaches claiming that the cosmos expansion can be explained as an emergent phenomenon [18,34-46]. Two key points in these approaches are the definition of energy and the form of the energymomentum conservation law [14, 19,34-38], and indeed their results are so sensitive to the energy definitions that have been employed [19].
In order to make the discussion clearer, consider a flat FRW universe with scale factor $a(t)$ [1]

$$
d s^{2}=-d t^{2}+a^{2}(t)\left[d r^{2}+r^{2} d \Omega^{2}\right]
$$

filled by a prefect fluid source with energy density $\rho$ and pressure $p$. Einstein field equations and energy-momentum conservation law (or equally the Bianchi identity) lead to

$$
\begin{aligned}
H^{2} & =\frac{8 \pi}{3} \rho, \\
3 H^{2}+2 \dot{H} & =-8 \pi p, \\
\dot{\rho}+3 H(\rho+p) & =0,
\end{aligned}
$$

forming the cornerstone of standard cosmology. Although, the third equation (OCL) is in full agreement with our observations on the cosmic fluid in matter and radiation dominated eras [1,47], its validity for the current accelerated cosmos is questionable [48], which may encourage us to consider the relaxed types of OCL to describe the current universe $[18,49]$. Combining the Friedmann equations with each other, we get

$$
\dot{H}=-4 \pi(\rho+p) \text {, }
$$


which can finally be added to the time derivative of the first Friedmann equation to reach OCL. Therefore, although the third equation of (2) is generally obtained from the conservation law $\left(T_{\mu \nu}^{; \nu}=0\right)$, one can easily find it by only using the Friedmann equations, a result which is the direct consequence of the Einstein field equations satisfying the Bianchi identity. It means that two equations of the above set of equations (2) are indeed enough to study the cosmos, and the third equation will be automatically valid and inevitable in this situation.

From the viewpoint of thermodynamics, if OCL is valid, then by applying the thermodynamics laws to the apparent horizon, as the proper causal boundary located at [2-4]

$$
\tilde{r}_{A}=a(t) r_{A}=\frac{1}{H},
$$

where $r_{A}$ is the comoving radius of apparent horizon, one can reach the Friedmann equations (2) [5-18]. Indeed, the validity of OCL is crucial, and its relaxation leads to modification of the Friedmann equations $[14-16,18]$. These results propose that the cosmos is so close to its thermodynamic equilibrium state [19-28], motivating us to be more confident of thermodynamical quantities, such as pressure, to study the cosmos [19].

For a FRW background filled by a fluid whose energy density $\rho$ is at most a function of time, we may reach

$$
E=\int T_{\alpha \beta} u^{\alpha} u^{\beta} d V=\int \rho d V=\rho V
$$

as the total energy confined to the volume $V$ and felt by a comoving observer with four velocities $u^{\alpha}=\delta_{t}^{\alpha}$. Equation (5) is fully compatible with the combination of the MisnerSharp mass, thermodynamics laws, and standard cosmology (2) [5-18].

Now, if we write $V=V_{0} a^{3}$, where $V_{0}$ is the comoving volume [1], using (5) and the thermodynamic pressure definition

$$
p=-\left(\frac{\partial E}{\partial V}\right)
$$

then we easily get OCL. In fact, it shows that the form of the conservation law depends on the energy definition. The above arguments also motivated the authors of [19] to use various energy definitions such as the Komar mass [50, 51] and

$$
\begin{aligned}
H^{2} & =\frac{8 \pi}{3} \rho, \\
3 H^{2}+2 \dot{H} & =8 \pi\left(\frac{\partial E}{\partial V}\right), \\
\dot{\rho}+3 H(\rho+p) & =0,
\end{aligned}
$$

instead of (2), in order to study the relations between energy definitions, thermodynamics laws, and the cosmic evolution, which led to a model for the cosmic fluid consistent with observations [19].
The Einstein field equations also admit another mass definition, namely, the Komar mass [47, 50, 51]:

$$
\mathscr{M}=2 \int\left(T_{\mu \nu}-\frac{1}{2} T g_{\mu \nu}\right) u^{\mu} u^{\nu} d V=(\rho+3 p) V
$$

which clearly differs from (5) unless we have a pressureless fluid $[41,47]$. Although this energy definition has originally been obtained by using the Einstein field equations [47], it is in accordance with various thermodynamic and holographic approaches [34-46]. In fact, if we accept this energy definition as a basic equation and not a secondary equation [3437], then there are various thermodynamic and holographic approaches employed to get the Friedmann and gravitational field equations in various theories by using the Komar mass definition [34-46].

Now, inserting the Komar energy definition into (6) and using $V=V_{0} a^{3}$, one can easily reach a new conservation law as

$$
\dot{\rho}+3(\dot{p}+3 H p)+3 H(\rho+p)=0,
$$

met by Komar mass, and we call it the Komar conservation law (KCL). For the pressureless systems, everything is consistent due to the fact that the above addressed energy definitions are equal. Similar types of this energy-momentum conservation law have also been obtained in theories which include a nonminimal coupling between the geometry and matter fields [14, 49, 52].

One basic consequence of the Komar mass is that not only $\rho$, but both of $\rho$ and $p$ participate in building the system energy which leads to appropriate models for dark sectors of cosmos, if one uses (7) to model the cosmos [19]. On the other hand, (9) claims that if we use the Komar mass, then OCL and thus (7) are not valid and other modified Friedmann equations should be employed to describe the cosmos.

In the general relativity (GR) framework, where $G_{\mu \nu}=$ $8 \pi T_{\mu \nu}$, the satisfaction of BI by the Einstein tensor $\left(G_{\mu}^{\nu}\right)$ is equivalent to the satisfaction of OCL by $T_{\mu}^{\nu}$. We saw that the form of conservation law depends on the energy definition (6), and indeed, if the Komar mass notion is used, then one can easily find that $T_{\mu}^{\nu}$ should satisfy KCL instead of OCL. Thus, in the GR framework, there is an inconsistency between the notions of BI (or equally OCL) and the Komar mass. This inconsistency together with the results of recent observations [48], admitting the break-down of OCL in the current accelerated era, motivates us to modify GR and, thus, the Friedmann equations by modifying the matter side of the field equations in the manner in which the modified matter part meets OCL, in agreement with the satisfaction of BI by the geometrical part. In summary, we think that OCL is very restrictive constraint on $T_{\mu}^{\nu}$. Probably, OCL should not be applied to the whole of $T_{\mu}^{\nu}$, and it should only be applied to some parts of $T_{\mu}^{\nu}$, the parts which may modify GR in a compatible way with the current accelerated cosmos [18, 48, 49].

There is also an elegant consistency between (5) and (2); i.e., $\rho$ is the energy density in (5), and it also appears in the 
first Friedmann equation. On the other hand, (8) implies that the quantity $(\rho+3 p)$ plays the role of energy density, while it is not present in the first Friedmann equation. In fact, if we define $\rho_{e} \equiv \rho+3 p$, then (9) can be rewritten as

$$
\dot{\rho}_{e}+3 H\left(\rho_{e}+p\right)=0
$$

equivalent to a hypothetical fluid with an effective energymomentum tensor of $T_{\mu}^{\nu}(e)=\operatorname{diag}\left(-\rho_{e}, p, p, p\right)$, meeting OCL (see [53] for a debate on effective energy-momentum tensor). In this manner, (5) for $T_{\mu}^{\nu}(e)$ will be equal to (8) for $T_{\mu}^{\nu}$. Indeed, the above arguments (specially (9)) claim that, even in the Einstein framework, OCL (2) may not be valid, if Komar mass is employed. In this situation, because OCL is the backbone of the Friedmann equations, we may conclude that the Friedmann equations (2) should be modified.

In summary, (i) the energy definition and the form of energy-momentum conservation law play crucial roles in getting the Friedmann equations in various theories [5-19]. (ii) We also showed that the form of energy-momentum conservation law depends on the energy definition, and if one uses the Komar mass, then KCL is obtained instead of OCL. (iii) The Komar mass is the backbone of some holographic and thermodynamic attempts to obtain field equations of various gravitational theories [34-46] motivating us to use KCL instead of OCL. (iv) Observational data admit the break-down of OCL in the current accelerated era [48]. It also motivates us to use the relaxed forms of OCL in order to describe the current universe. On the other hand, there are also various models for the current accelerated universe satisfying OCL [1]. Therefore, it seems that some inconsistency exists between the various definitions of energy, continuity, and Friedmann equations. One may conclude that the fluid obtained from the observation may not be the real cosmic fluid, and it indeed represents some less well-known or even unknown parts of the energy source which become important, effective, and tangible in the current cosmos. We want to say that the real cosmic fluid may satisfy conservation laws such as KCL instead of OCL, while some of its parts, responsible for the current accelerated expansion, may satisfy OCL.

As we mentioned, based on (8), one may conclude that pressure has some contribution to the energy density of system. The question that arises here is, what if this contribution can describe the current accelerated universe? In other words, are there combinations of $\rho$ and $p$ of a baryonic source which can play the role of dark energy? Here, we are going to find out the answers of the mentioned questions by studying some consequences of accepting (6), the Komar mass definition, and thus (9), in cosmological setups. In the next section, using thermodynamics laws, Bekenstein entropy, and (9), we address some models for dark energy. The third section is devoted to a summary and concluding remarks. Throughout this work, the unit of $c=$ $\hbar=G=k_{B}=1$, where $k_{B}$ denotes the Boltzmann constant, has been employed.

\section{Komar Definition of Energy and the Friedmann Equations}

For an energy-momentum source with $T_{a}^{b}=\operatorname{diag}(-\rho, p, p$, $p)$, the amount of energy crossing the apparent horizon is evaluated as [8]

$$
\delta Q^{m}=A\left(T_{a}^{b} \partial_{b} \widetilde{r}_{A}+W \partial_{a} \tilde{r}_{A}\right) d x^{a},
$$

where $A=4 \pi \widetilde{r}_{A}^{2}=A_{0} a^{2}$ and $W=(\rho-p) / 2$ denote the horizon area and the work density, respectively. $A_{0}=4 \pi r_{A}^{2}$ is also the comoving area. After some calculations, one obtains $[8,14-17]$

$$
\delta Q^{m}=-3 V H(\rho+p) d t
$$

combined with the Clausius relation $\left(T d S_{A}=-\delta Q^{m}\right)$, to get $[8,15]$

$$
3 V H(\rho+p) d t=\frac{H}{2 \pi} d S_{A}
$$

where the additional minus sign in the Clausius relation is due to the direction of energy flux $[8,15]$. Here, $S_{A}$ is the horizon entropy and the Cai-Kim temperature $(T=H / 2 \pi)$ is used to reach this equation $[8,15]$. The role of energymomentum conservation law in deriving the Friedmann equations is now completely clear. The effects of considering various approximations and temperatures have been studied in [15].

2.1. Modified Friedmann Equations. Here, using some simple examples, we are going to show the effects of considering KCL in modifying the standard cosmology.

Case (i). If OCL is valid, then (13) is reduced to

$$
d \rho=-\frac{3 H^{4}}{8 \pi^{2}} d S_{A}
$$

covering the first Friedmann equation whenever $S=A / 4$. In this situation, (9) implies that

$$
\begin{aligned}
\dot{p}+3 H p & =0 \longrightarrow \\
p(a) & =p_{0} a^{-3},
\end{aligned}
$$

where $p_{0}$ is the integration constant and we considered $a_{0}=1$ ( $a_{0}$ is the current value of the scale factor). In this manner, for $S_{A}=A / 4$, we reach

$$
\begin{aligned}
H^{2} & =\frac{8 \pi}{3} \rho, \\
3 H^{2}+2 \dot{H} & =-8 \pi p_{0} a^{-3}, \\
\dot{\rho}+3 H\left(\rho+p_{0} a^{-3}\right) & =0,
\end{aligned}
$$

instead of (2). It is easy to check that for power law regimes in which $\rho \propto a^{\beta}$, conservation law leads to $p_{0}=0$ and $\beta=-3$, which is nothing but the dust source, in full agreement with our previous results indicating that for a pressureless source 
everything is consistent. The geometrical parts of the two first equations of (16) lead us to the Einstein tensor. It is in fact the direct result of attributing the Bekenstein entropy to the horizon [8]. Therefore, mathematically, since the Einstein tensor obeys $\mathrm{BI}$, the appearing $\rho$ and $p$ should also satisfy OCL, an expectation in full agreement with (15) and thus the third line of (16).

However, the general solution of the obtained conservation law (16) is

$$
\rho(a)=a^{-3}\left[c-3 p_{0} \ln (a)\right],
$$

where $c$ is the integration constant. It clearly covers the ordinary matter era whenever $p_{0}=0$. From (15) and (17), we reach

$$
\begin{aligned}
& p=p_{0}, \\
& \rho=c,
\end{aligned}
$$

for the current era. Thus, if we have $p_{0}=w_{0} c$ and $c=$ $\Lambda>0$, where $\Lambda$ and $w_{0}$ denote the current values of the energy density and the equation of state (EoS) of dark energy, respectively, then the obtained fluid may theoretically generate the current accelerating universe whenever $w_{0} \leq$ $-2 / 3$. Now, we can write

$$
\begin{aligned}
& p(a)=w_{0} \Lambda a^{-3}, \\
& \rho(a)=\Lambda a^{-3}\left[1-3 w_{0} \ln (a)\right] .
\end{aligned}
$$

In this manner, $\operatorname{EoS}(w)$ and the deceleration parameter $(q)$ are evaluated as

$$
\begin{aligned}
& w \equiv \frac{p}{\rho}=\frac{w_{0}}{1-3 w_{0} \ln (a)}, \\
& q \equiv-1-\frac{\dot{H}}{H^{2}}=\frac{1}{2}\left(1+\frac{3 w_{0}}{1-3 w_{0} \ln (a)}\right) .
\end{aligned}
$$

At the $a \longrightarrow 0$ limit, independently of the value of $w_{0}$, we have $w \longrightarrow 0$ and $q \longrightarrow 1 / 2$. Moreover, one can easily see that, at the $a \longrightarrow 1$ limit, the consistent values with observations for $w$ and $q$ are also obtainable, depending on the value of $w_{0}$. Despite this compatibility, there is a singularity at the behavior of $q$ and $w$ for $w_{0}<0$, located at $a=e^{1 / 3 w_{0}}$, meaning that this solution may be at most useable to study the $a>e^{1 / 3 w_{0}}$ era. However, as it is obvious from Figure 1 , it does not lead to suitable $q$ and $w$ even for $a>e^{1 / 3 w_{0}}$. Therefore, as we previously saw, only the $w_{0}=0$ case can meet all of the desired expectations; i.e., it is in agreement with the Friedmann equations, which both addressed energy definitions and thus the matter dominated era.

Case (ii). If $T_{\mu}^{\nu}(e)$, satisfying (10), is used instead of $T_{\mu}^{\nu}$ in order to evaluate $\delta Q^{m}$ (11) and we follow the Cai-Kim approach $[8,14-18]$, then some calculations lead to

$$
\begin{aligned}
H^{2} & =\frac{8 \pi}{3} \rho_{e}, \\
3 H^{2}+2 \dot{H} & =-8 \pi p, \\
\dot{\rho}_{e}+3 H\left(\rho_{e}+p\right) & =0,
\end{aligned}
$$



FIGURE 1: Here, $w_{0}=-1$, dot and solid lines represent $w$ and $q$, respectively.

whenever $S_{A}=A / 4$. These equations would also be obtainable by modifying the Einstein field equations as $G_{\mu \nu}=8 \pi T_{\mu \nu}(e)$, in agreement with attributing the Bekenstein entropy to the apparent horizon (see the paragraph after (16)). Now, considering the simple case $w_{e} \equiv p / \rho_{e}$ and using the above equations, we can easily find $w_{e}=w /(1+3 w)$. This result indicates that a baryonic source, with $w>0$, cannot lead to a hypothetical fluid with $w_{e}<0$ in cosmos, meaning that the obtained modified Friedmann equations cannot describe the current accelerated universe.

Case (iii). Now, bearing (9) in mind and defining an effective pressure as $p_{e}=\beta p$, one reaches

$$
\dot{\rho}+\frac{3}{\beta}\left[\dot{p}_{e}+(4-\beta) H p_{e}\right]+3 H\left(\rho+p_{e}\right)=0,
$$

reduced to

$$
\dot{\rho}+3 H\left(\rho+p_{e}\right)=0,
$$

whenever we have

$$
\begin{aligned}
\dot{p}_{e}+(4-\beta) H p_{e} & =0 \Longrightarrow \\
p_{e} & =p_{e}^{0} a^{\beta-4},
\end{aligned}
$$

where $p_{e}^{0}$ is the integration constant. Inserting this result into (23), it is easy to obtain

$$
\rho=\frac{c}{a^{3}}-3 \frac{p_{e}^{0}}{\beta-1} a^{\beta-4} .
$$

Here, $c$ is an integration constant, and it is worthwhile to mention that the ordinary matter and radiation dominated eras are recovered at the limits of $p_{e}^{0}=0$ and $c, \beta=$ 0 , respectively, meaning that the obtained fluid can unify these eras into one model. In this manner, we can argue 
that although $T_{\mu \nu}$ is the true energy-momentum tensor, an effective tensor of $T_{\mu}^{v}(e)=\operatorname{diag}\left(-\rho, p_{e}, p_{e}, p_{e}\right)$ should be considered, which may lead us to a modified gravitational equation of the form $G_{\mu \nu}=8 \pi T_{\mu \nu}(e)$. Now, combining (24) and (25) with each other, one finds

$$
w_{e} \equiv \frac{p_{e}}{\rho}=\beta w=\frac{(\beta-1) p_{e}^{0} a^{3}}{(\beta-1) c a^{4-\beta}-3 p_{e}^{0} a^{3}} .
$$

For $\beta>1$ and $\beta<1$, we have $w_{e} \longrightarrow 0$ and $w_{e} \longrightarrow(1-\beta) / 3$, respectively, at the $a \longrightarrow 0$ limit. Moreover, independently of the value of $\beta$, one can see that $w_{e} \longrightarrow(\beta-1) p_{e}^{0} /((\beta-1) c-$ $\left.3 p_{e}^{0}\right) \equiv w_{0}$, leading to $c=p_{e}^{0}\left(\beta-1+3 w_{0}\right) /(\beta-1) w_{0}$, whenever $a \longrightarrow 1$. Using the latest result and the $1+z=a^{-1}$ relation, where $z$ is the redshift, we can finally rewrite (26) as

$$
w_{e}=\beta w=\frac{w_{0}(\beta-1)}{\left(\beta-1+3 w_{0}\right)(1+z)^{\beta-1}-3 w_{0}} .
$$

If $w_{0}<0$ and $c>0$, then for $\beta>1-3 w_{0}$ and $p_{e}^{0}<0$ (or equally $\left.p_{e}<0\right)$ we have $\rho>0, p<0$, and $w_{e}(z \gg 1) \longrightarrow 0$ meaning that this fluid may describe the universe history from the matter dominated era to the current accelerating phase. In this manner, if we either modify Einstein equations as $G_{\mu \nu}=8 \pi T_{\mu \nu}(e)$ or follow the Cai-Kim recipe by using $T_{\mu \nu}(e)=\operatorname{diag}\left(-\rho, p_{e}, p_{e}, p_{e}\right)$, then we obtain

$$
\begin{aligned}
H^{2} & =\frac{8 \pi}{3} \rho, \\
3 H^{2}+2 \dot{H} & =-8 \pi p_{e}, \\
\dot{\rho}+3 H\left(\rho+p_{e}\right) & =0 .
\end{aligned}
$$

As before, two first equations imply that $T_{\mu \nu}(e)$ should satisfy OCL, in full agreement with (23), and thus the third line of (28). Calculations for the deceleration parameter lead to

$$
q \equiv-1-\frac{\dot{H}}{H^{2}}=\frac{1}{2}\left(1+3 w_{e}\right) .
$$

Suitable values of $\beta$ should be found by considering the transition point at which $q_{t}=0$ and $w_{e}=-1 / 3 \equiv w_{e}^{t}$. In addition, simple calculations for the transition redshift lead to

$$
z_{t}=-1+\left(\frac{\beta-1+3 w_{0}}{3 w_{0}(2-\beta)}\right)^{1 /(1-\beta)},
$$

indicating that $\beta$ should also meet the $\beta>2$ condition to reach positive meaningful solutions for $z_{t}$. Because $w_{0}<$ $w_{e}^{t}$, we have the relation $1-3 w_{0}>2$ meaning that the $\beta>2$ condition is in agreement with the previously obtained condition $\left(\beta>1-3 w_{0}\right)$. Besides, although we can obtain suitable values of $z_{t}$ for $1<\beta<1-3 w_{0}$, in this manner, negative amounts will be attained by $\rho$ which finally reject this case. For other values of $\beta$, energy density will grow much faster than the matter density, proportional to $(1+z)^{3}$, by increasing $z$. Thus, only the $\beta>1-3 w_{0}$

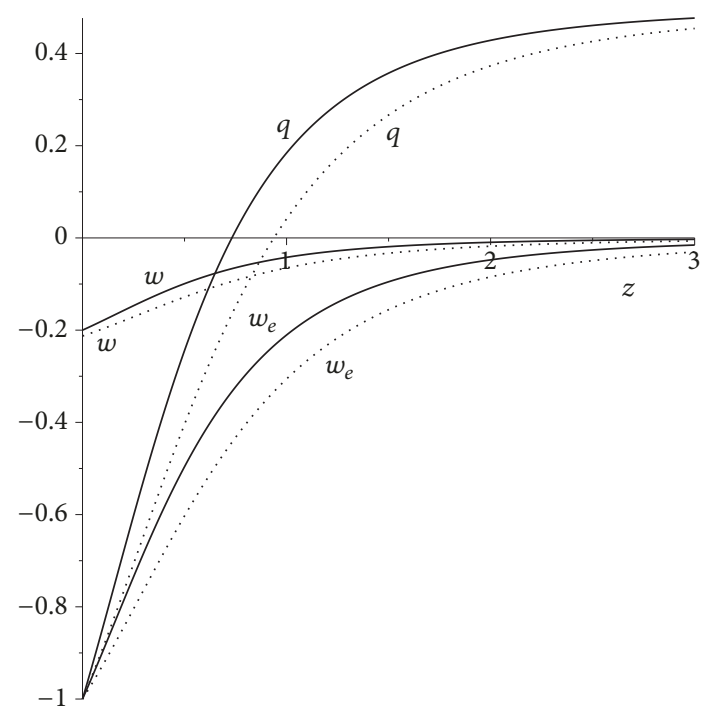

FIGURE 2: $w_{e}, w$, and $q$ versus $z$ when $w_{0}=-1$. Solid lines: $\beta=5$. Dot lines: $\beta=4.7$.

case can be meaningful. In Figure 2, deceleration parameter, $w_{e}$ and $w$ have been plotted versus $z$ for some values of $\beta$.

Hence, if we accept both the Komar mass definition and the thermodynamic pressure notion, we can modify the standard cosmology as (28) in which the solutions of the field equations can theoretically unify both the matter dominated era and the current accelerated universe. However, in this manner, since $\beta>0$, the original source has a negative pressure $\left(p=p_{e} / \beta<0\right)$. Therefore, it is useful to emphasize that, despite the proper obtained results, since $p<0$, this approach does not lead to a baryonic model for the energy source during the cosmos evolution, i.e., a model with $0 \leq$ $p \leq(1 / 3) \rho$.

Case (iv). As another case, bearing (9) in mind, defining $p_{e}=\beta p, \rho_{e}=\rho+\alpha p$, where $\alpha$ and $\beta$ are unknown constants that should meet the $\alpha+\beta=4$ condition, and considering the $\dot{p}=\dot{p}_{e}=0$ case, one can follow the above recipes to obtain

$$
\begin{aligned}
H^{2} & =\frac{8 \pi}{3} \rho_{e}, \\
3 H^{2}+2 \dot{H} & =-8 \pi p_{e}, \\
q & =\frac{1}{2}\left(1+\frac{3 p_{e}}{\rho_{e}}\right), \\
\dot{\rho}_{e}+3 H\left(\rho_{e}+p_{e}\right) & =0 \longrightarrow \\
\rho_{e} & =\left(\rho_{e}^{0}+\beta p\right)(1+z)^{3}-\beta p,
\end{aligned}
$$

where $\rho_{e}^{0}$ is the integration constant. As the previous cases, since the rhs of the two first equations are nothing but the Einstein tensor meeting BI, $\rho_{e}$ and $p_{e}$ should satisfy OCL (or equally the last line). Indeed, since we have $\dot{p}_{e}=0$, the 
$\alpha+\beta=4$ constraint is unavoidable only if we want KCL to be reduced to OCL (the last line). We finally reach

$$
\begin{aligned}
\rho & =\left(\rho_{e}^{0}+\beta p\right)(1+z)^{3}-4 p, \\
w & \equiv \frac{p}{\rho}=\frac{p}{\left(\rho_{e}^{0}+\beta p\right)(1+z)^{3}-4 p}, \\
w_{e} & \equiv \frac{p_{e}}{\rho_{e}}=\frac{\beta p}{\left(\rho_{e}^{0}+\beta p\right)(1+z)^{3}-\beta p} .
\end{aligned}
$$

As we know, the current accelerated universe $(z=0)$ implies $p_{e}<0$ and $w_{e}(z=0) \equiv w_{0} \leq-2 / 3$ (or equally $\rho_{e}(z=$ $\left.0)>(2 / 3)\left(-p_{e}\right)\right)[1]$. Thus, the above results admit a fluid with positive pressure whenever $\beta<0$. In this manner, for $z \geq 0$, if $\rho_{e}^{0}>\alpha p$, satisfied when $\beta<\alpha w_{0}$ leading to $\beta<4 w_{0} /\left(1+w_{0}\right)$ (or equally $4<\alpha\left(1+w_{0}\right)$ ), then we have $\rho_{e}, \rho>0$. This result indicates that we should have $w_{0}>-1$, in agreement with some observations [1], to meet the $\beta<0$ condition. For this case, while, unlike $w, w_{e}$ is always negative, both $w$ and $w_{e}$ approach zero for $z \gg 1$. Using the above results, we finally get

$$
\begin{aligned}
w & =\frac{w_{0}}{\beta\left(w_{0}+1\right)(1+z)^{3}-4 w_{0}}, \\
w_{e} & =\frac{w_{0}}{\left(w_{0}+1\right)(1+z)^{3}-w_{0}}, \\
q & =\frac{\left(w_{0}+1\right)(1+z)^{3}+2 w_{0}}{2\left[\left(w_{0}+1\right)(1+z)^{3}-w_{0}\right]},
\end{aligned}
$$

leading to $z_{t}=-1+\left(-2 w_{0} /\left(1+w_{0}\right)\right)^{1 / 3}$ for the transition redshift. The parameters $w, w_{e}$, and $q$ have been plotted in Figures 3 and 4 for $w_{0}=-0 \cdot 73$ [1]. It is also worth remembering that the current observational data on dark energy density and pressure in fact give us the corresponding values of $\rho_{e}$ and $p_{e}$. As is apparent, there are some values of $\beta$ (and thus $\alpha$ ) for which the maximum value of $w$ is at most equal to $1 / 3$, signalling us to a baryonic source (since $\beta<0$ and $w>0$, we have $p>0$ and $\rho>0$ ). Therefore, $w_{e}$ and $q$ display proper behavior, meaning that it is mathematically enough to only consider some parts of $T_{\mu \nu}$, represented by $T_{\mu \nu}(e)$, to modify the standard cosmology as (31) (or equally the Einstein field equations as $G_{\mu \nu}=8 \pi T_{\mu \nu}(e)$ ) which gives us a suitable description for the current phase of the universe.

\section{Conclusion}

Mach principle states that geometry inherits its properties from inside it; i.e., the geometrical information is related to the energy of the source. Hence, it does not limit us to certain information for the energy source. This principle along with OCL and BI is the backbone of Einstein theory and thus, standard cosmology, in full agreement with the thermodynamics laws. Based on this theory, the Einstein $\left(G_{\mu \nu}\right)$ and energy-momentum $\left(T_{\mu \nu}\right)$ tensors are in a direct relation as $G_{\mu \nu}=8 \pi T_{\mu v}$. Hence, by having the whole information of the matter source $\left(T_{\mu \nu}\right)$, one can find $G_{\mu \nu}$ and

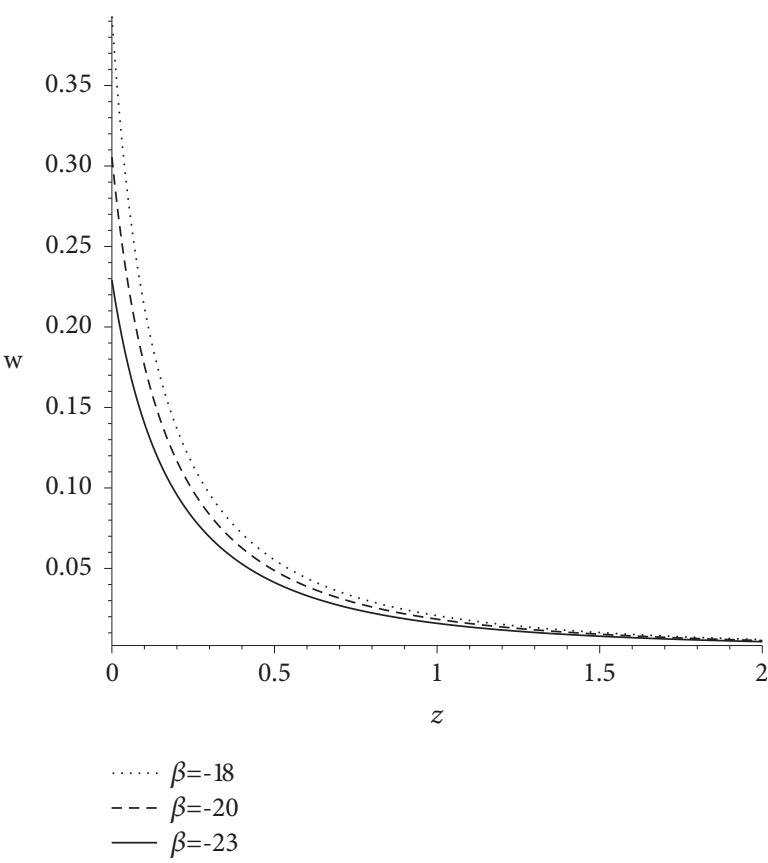

FIGURE 3: $w$ has been depicted versus $z$ for some values of $\beta$.

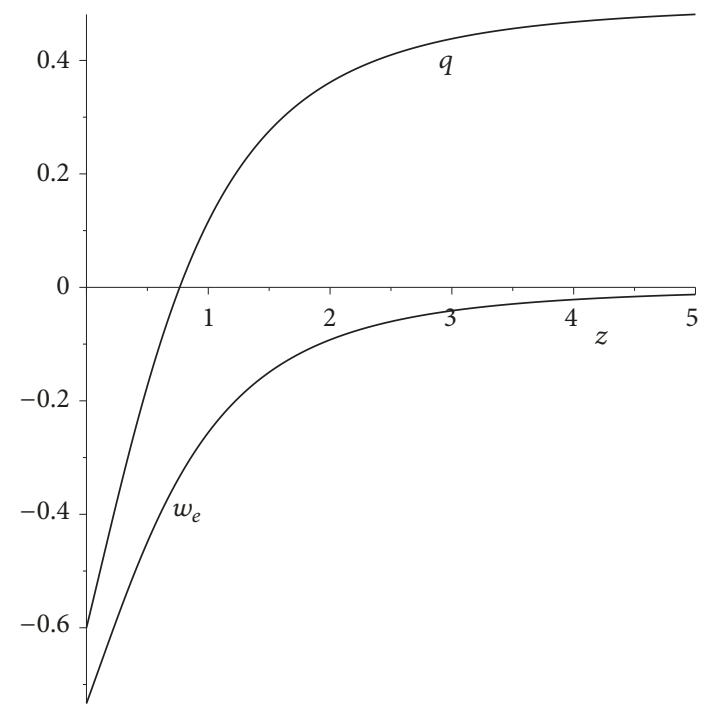

FIGURE 4: $w_{e}$ and $q$ versus $z$.

then the space-time metric which finally gives us the whole information of geometry. It is also worth mentioning that the recent observation admits the break-down of OCL in the current accelerated era [48].

Besides, there are various approaches to gravity and cosmology in which the Komar definition of energy is their foundation. Here, by accepting this energy definition and using the thermodynamic pressure definition, a relaxed energymomentum conservation law (KCL) has been obtained, helping us in getting some modified energy-momentum tensors $\left(T_{\mu \nu}(e)\right)$ satisfying OCL. Consequently, applying the thermodynamics laws to the apparent horizon and attributing 
the Cai-Kim temperature to it, we could find out some simple modified Friedmann equations. Our results are in fact equal to modifying the Einstein field equations as $G_{\mu \nu}=8 \pi T_{\mu \nu}(e)$. Therefore, in agreement with the satisfaction of BI by the Einstein tensor, $T_{\mu \nu}(e)$ is also meeting OCL. The study shows that an appropriate choice of $T_{\mu \nu}(e)$ allows us to unify the dark sectors of cosmos into one model. In fact, the analysis of the last case $(i v)$ confirms that if suitable $T_{\mu \nu}(e)$ is used instead of $T_{\mu \nu}$ in order to modify the standard cosmology (31), then $T_{\mu \nu}(e)$ can mathematically describe the current accelerated cosmos, while $T_{\mu \nu}$ can display a baryonic source.

In summary, (i) $T_{\mu \nu}(e)$, made of $T_{\mu \nu}$, satisfies OCL (or equally $\nabla^{\mu} T_{\mu \nu}(e)=0$ ) and can describe the current accelerated universe. (ii) In this manner, $T_{\mu \nu}$, satisfying $\mathrm{KCL}$, can even show a baryonic behavior by itself. These results may be translated as follows: the less well-known or even unknown aspects of $T_{\mu \nu}$, represented by $T_{\mu \nu}(e)$, are responsible for the current accelerated phase of the universe. The origin and the emergence conditions of $T_{\mu \nu}(e)$ may be found out by studying this part in the framework of other physical theories such as quantum field theory.

\section{Data Availability}

There is no data used in this paper.

\section{Conflicts of Interest}

The authors declare that there are no conflicts of interest regarding the publication of this paper.

\section{Acknowledgments}

The work of H. Moradpour has been supported financially by Research Institute for Astronomy \& Astrophysics of Maragha (RIAAM). J. P. Morais Graça and I. P. Lobo are supported by Coordenação de Aperfeiçoamento de Pessoal de Nível Superior (CAPES).

\section{References}

[1] M. Roos, Introduction to Cosmology, John Wiley and Sons, UK, 2nd edition, 2003.

[2] S. A. Hayward, "Unified first law of black-hole dynamics and relativistic thermodynamics," Classical and Quantum Gravity, vol. 15, no. 10, p. 3147, 1998.

[3] S. A. Hayward, S. Mukohyana, and M. C. Ashworth, "Dynamic black-hole entropy," Physics Letters A, vol. 256, no. 5-6, pp. 347350, 1999.

[4] D. Bak and S. J. Rey, "Cosmic holography," Classical and Quantum Gravity, vol. 17, no. 15, pp. L83-L89, 2000.

[5] R.-G. Cai, "Cardy-Verlinde formula and thermodynamics of black holes in de Sitter spaces," Nuclear Physics B, vol. 628, no. 1-2, pp. 375-386, 2002.

[6] R.-G. Cai, "Cardy-Verlinde formula and asymptotically de Sitter spaces," Physics Letters B, vol. 525, no. 3-4, pp. 331-336, 2002.

[7] A. V. Frolov and L. Kofman, "Inflation and de Sitter thermodynamics," Journal of Cosmology and Astroparticle Physics, vol. 2003, no. 5, p. 9, 2003.
[8] R. G. Cai and S. P. Kim, "First Law of Thermodynamics and Friedmann Equations of Friedmann-Robertson-Walker Universe," Journal of High Energy Physics, vol. 2005, no. 2, p. 50, 2005.

[9] R. Whisker, "Transplanckian energy production and slow roll inflation," Physical Review D, vol. 71, Article ID 023516, 2005.

[10] R. S. Bousso, "Cosmology and the S matrix," Physical Review D: Particles, Fields, Gravitation and Cosmology, vol. 71, Article ID 064024, 2005.

[11] A. Sheykhi, B. Wang, and R. G. Cai, "Thermodynamical Properties of Apparent Horizon in Warped DGP Braneworld," Nuclear Physics B, vol. 779, no. 1, 2007.

[12] A. Sheykhi, B. Wang, and R.-G. Cai, "Deep connection between thermodynamics and gravity in Gauss-Bonnet braneworlds," Physical Review D: Particles, Fields, Gravitation and Cosmology, vol. 76, no. 2, Article ID 023515, 5 pages, 2007.

[13] D. W. Tian and I. Booth, "Apparent horizon and gravitational thermodynamics of the universe: solutions to the temperature and entropy confusions and extensions to modified gravity," Physical Review D: Particles, Fields, Gravitation and Cosmology, vol. 92, Article ID 024001, 2015.

[14] H. Moradpour, "Thermodynamics of flat FLRW universe in Rastall theory," Physics Letters B, vol. 757, pp. 187-191, 2016.

[15] H. Ebadi and H. Moradpour, "Thermodynamics of universe with a varying dark energy component," International Journal of Modern Physics D, vol. 24, no. 14, Article ID 1550098, 2015.

[16] H. Moradpour and R. Dehghani, "Thermodynamical study of FRW universe in quasi-topological theory," Advances in High Energy Physics, vol. 2016, Article ID 7248520, 10 pages, 2016.

[17] H. Moradpour, "Implications, consequences and interpretations of generalized entropy in the cosmological setups," International Journal of Theoretical Physics, vol. 55, no. 9, pp. 41764184, 2016.

[18] H. Moradpour, A. Bonilla, E. M. Abreu, and J. A. Neto, "Accelerated cosmos in a nonextensive setup," Physical Review D: Particles, Fields, Gravitation and Cosmology, vol. 96, no. 12, Article ID 123504, 2017.

[19] H. Moradpour, R. C. Nunes, E. M. Abreu, and J. A. Neto, "A note on the relations between thermodynamics, energy definitions and Friedmann equations," Modern Physics Letters A, vol. 32, no. 13, 1750078, 20 pages, 2017.

[20] H. Moradpour, "Implications, consequences and interpretations of generalized entropy in the cosmological setups," International Journal of Theoretical Physics, vol. 55, no. 268, 2016.

[21] H. Moradpour, A. Sheykhi, N. Riazi, and B. Wang, "Necessity of dark energy from thermodynamic arguments," Advances in High Energy Physics, vol. 2014, Article ID 718583, 9 pages, 2014.

[22] N. Radicella and D. Pavón, "Thermodynamical analysis on a braneworld scenario with curvature corrections," Physics Letters B, vol. 704, no. 4, pp. 260-264, 2011.

[23] N. Radicella and D. Pavón, "A thermodynamic motivation for dark energy," General Relativity and Gravitation, vol. 44, no. 3, pp. 685-702, 2012.

[24] D. Pavón and W. Zimdahl, "A thermodynamic characterization of future singularities?" Physics Letters B, vol. 708, no. 3-5, pp. 217-220, 2012.

[25] D. Pavón and N. Radicella, "Does the entropy of the universe tend to a maximum?" General Relativity and Gravitation, vol. 45, no. 1, pp. 63-68, 2013.

[26] D. Pavón, "Why the expansion of the Universe is accelerated," International Journal of Geometric Methods in Modern Physics, vol. 11, no. 2, Article ID 1460007, 16 pages, 2014. 
[27] J. P. Mimoso and D. Pavón, "Considerations on the thermal equilibrium between matter and the cosmic horizon," Physical Review D: Particles, Fields, Gravitation and Cosmology, vol. 94, no. 10, Article ID 103507, 2016.

[28] P. B. Krishna and T. K. Mathew, "Holographic equipartition and the maximization of entropy," Physical Review D: Particles, Fields, Gravitation and Cosmology, vol. 96, Article ID 063513, 2017.

[29] F. C. Santos, M. L. Bedran, and V. Soares, "On the thermodynamic stability of the modified Chaplygin gas," Physics Letters. B. Particle Physics, Nuclear Physics and Cosmology, vol. 646, no. 5-6, pp. 215-221, 2007.

[30] M. Bedran, V.Soares, and M. Araújo, “Temperature evolution of the FRW universe filled with modified Chaplygin gas," Physics Letters B, vol. 659, no. 3, pp. 462-465, 2008.

[31] M. L. Bedran and V. Soares, "Negative pressures in classical and cosmological fluids and their thermodynamic stability," Progress of Theoretical Physics, vol. 123, p. 1, 2010.

[32] E. M. Barboza, R. C. Nunes, E. M. Abreu, and J. A. Neto, "Thermodynamic aspects of dark energy fluids," Physical Review D: Particles, Fields, Gravitation and Cosmology, vol. 92, no. 8, Article ID 083526, 2015.

[33] G. Z. Babar, M. Jamil, and Y.-K. Lim, "Dynamics of a charged particle around a weakly magnetized naked singularity," International Journal of Modern Physics D, vol. 25, no. 2, Article ID 1650024, 2016.

[34] T. Padmanabhan, "Entropy of static spacetimes and microscopic density of states," Classical and Quantum Gravity, vol. 21, no. 18, pp. 4485-4494, 2004.

[35] T. Padmanabhan, "Equipartition of energy in the horizon degrees of freedom and the emergence of gravity," Modern Physics Letters A, vol. 25, no. 14, pp. 1129-1136, 2010.

[36] R.-G. Cai, L.-M. Cao, and N. Ohta, "Friedmann equations from entropic force," Physical Review D: Particles, Fields, Gravitation and Cosmology, vol. 81, no. 6, Article ID 061501, 4 pages, 2010.

[37] E. Verlinde, Journal of High Energy Physics (JHEP), vol. 2011, no. 04, p. 29, 2011.

[38] T. Padmanabhan, "Emergence and Expansion of Cosmic Space as due to the Quest for Holographic Equipartition," https:// arxiv.org/abs/1206.4916.

[39] Y. Ling, J. P. Wu, and J. Cosmol, "A note on entropic force and brane cosmology," Astroparticle Physics, vol. 1008, p. 017, 2010.

[40] A. Sheykhi, "Modified Friedmann equations on the brane from entropic," International Journal of Theoretical Physics, vol. 51, p. 185, 2012.

[41] A. Sheykhi, H. Moradpour, and N. Riazi, "Lovelock gravity from entropic force," General Relativity and Gravitation, vol. 45, no. 5, pp. 1033-1049, 2013.

[42] A. Sheykhi, M. H. Dehghani, and S. E. Hosseini, "Friedmann equations in braneworld scenarios from emergence of cosmic space," Physics Letters B, vol. 726, no. 1-3, pp. 23-27, 2013.

[43] H. Moradpour and A. Sheykhi, "From the Komar mass and entropic force scenarios to the Einstein field equations on the Ads brane," International Journal of Theoretical Physics, vol. 55, no. 2, pp. 1145-1155, 2016.

[44] H. Moradpour, A. Amiri, and A. Sheykhi, "Implications of maximum acceleration on dynamics," Iranian Journal of Science and Technology, Transactions A: Science, pp. 1-7, 2018.

[45] N. Komatsu, "Cosmological model from the holographic equipartition law with a modified Rényi entropy," The European Physical Journal C, vol. 77, p. 229, 2017.
[46] H. Moradpour, A. Sheykhi, C. Corda, and I. G. Salako, "Implications of the generalized entropy formalisms on the Newtonian gravity and dynamics," Physics Letters B, vol. 783, pp. 82-85, 2018.

[47] S. Weinberg, Gravitation and Cosmology, Wiley, New York, NY, USA, 1972.

[48] T. Josset and A. Perez, "Dark energy from violation of energy conservation," Physical Review Letters, vol. 118, Article ID 021102, 2017.

[49] H. Moradpour, Y. Heydarzade, F. Darabi, and I. G. Salako, "A generalization to the Rastall theory and cosmic eras," The European Physical Journal C, vol. 77, no. 4, 2017.

[50] A. Komar, "Covariant conservation laws in general relativity," Physical Review A: Atomic, Molecular and Optical Physics, vol. 113, pp. 934-936, 1959.

[51] A. Komar, "Positive-Definite Energy Density and Global Consequences for General Relativity," Physical Review A: Atomic, Molecular and Optical Physics, vol. 129, no. 4, pp. 1873-1876, 1963.

[52] P. Rastall, "Generalization of the Einstein theory," Physical Review D: Particles, Fields, Gravitation and Cosmology, vol. 6, pp. 3357-3359, 1972.

[53] F. Darabi, H. Moradpour, I. Licata, Y. Heydarzade, and C. Corda, "Einstein and Rastall theories of gravitation in comparison," The European Physical Journal C, vol. 78, no. 1, p. 25, 2018. 



Submit your manuscripts at

www.hindawi.com

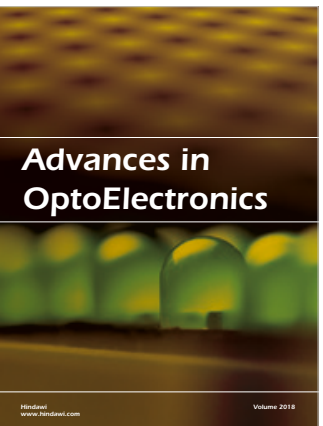

\section{Rotcting Machinery}
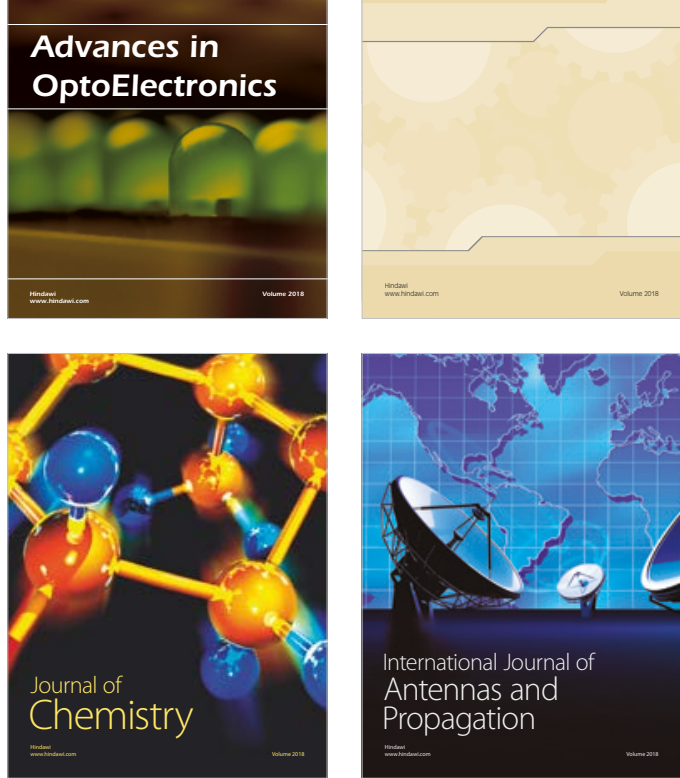

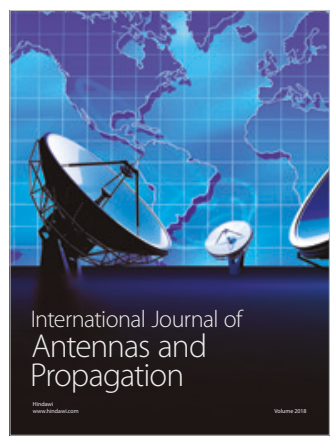

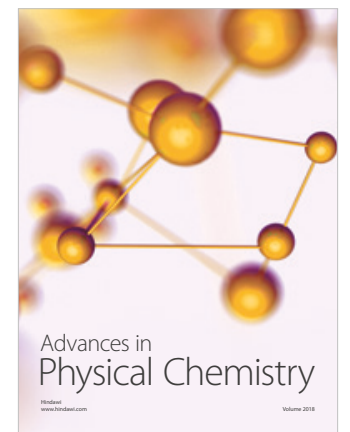

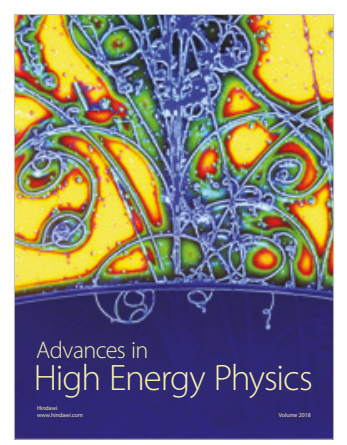

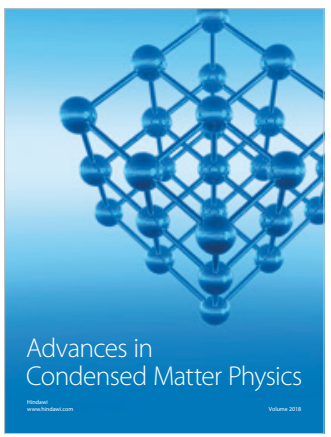

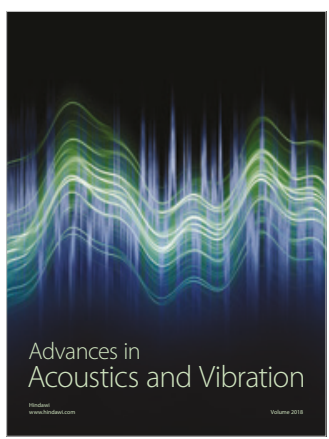

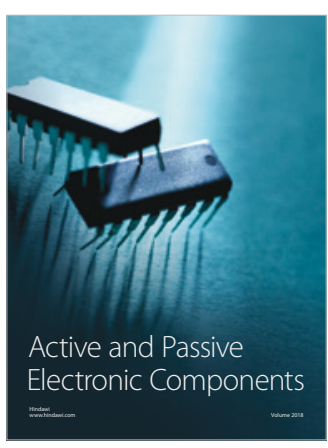
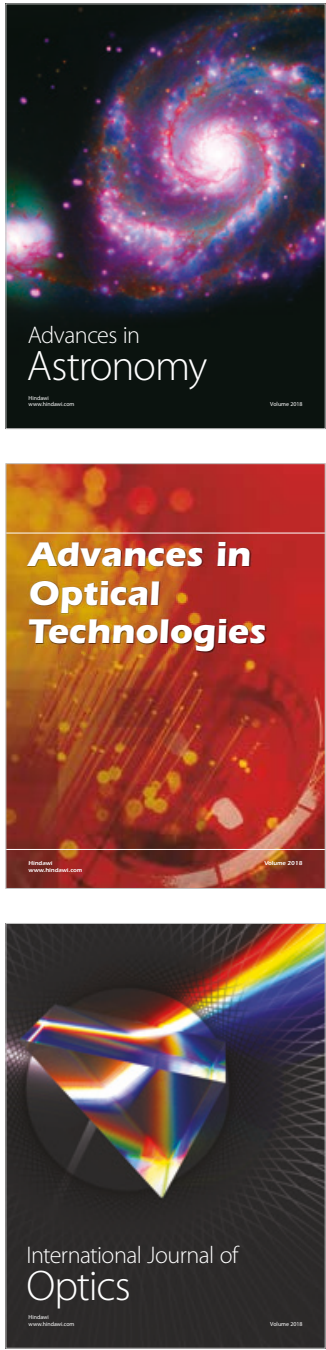